\title{
An Algorithm for the Factorization of Split Quaternion Polynomials
}

\author{
Daniel F. Scharler*® and Hans-Peter Schröcker
}

\begin{abstract}
We present an algorithm to compute all factorizations into linear factors of univariate polynomials over the split quaternions, provided such a factorization exists. Failure of the algorithm is equivalent to non-factorizability for which we present also geometric interpretations in terms of rulings on the quadric of non-invertible split quaternions. However, suitable real polynomial multiples of split quaternion polynomials can still be factorized and we describe how to find these real polynomials. Split quaternion polynomials describe rational motions in the hyperbolic plane. Factorization with linear factors corresponds to the decomposition of the rational motion into hyperbolic rotations. Since multiplication with a real polynomial does not change the motion, this decomposition is always possible. Some of our ideas can be transferred to the factorization theory of motion polynomials. These are polynomials over the dual quaternions with real norm polynomial and they describe rational motions in Euclidean kinematics. We transfer techniques developed for split quaternions to compute new factorizations of certain dual quaternion polynomials.
\end{abstract}

Mathematics Subject Classification. 16S36, 12D05, 51M09, 51M10, 70B10.

Keywords. Skew polynomial ring, Null quadric, Clifford translation, Left/right ruling, Zero divisor, Hyperbolic kinematics.

\section{Introduction}

In kinematics, robotics and mechanism science Hamiltonian quaternions and dual quaternions have been employed to parametrize the group of Euclidean

This article is part of the ENGAGE 2019 Topical Collection on Geometric Algebra for Computing, Graphics and Engineering edited by Linwang Yuan (EiC), Werner Benger, Dietmar Hildenbrand, and Eckhard Hitzer.

*Corresponding author. 
displacements $\mathrm{SE}(3)$ as well as its subgroups $\mathrm{SO}(3)$ and $\mathrm{SE}(2)$. The rich algebraic structure of the quaternion models allow to investigate certain problems from an algebraic point of view. Rational motions can be represented by polynomials over the ring of quaternions or dual quaternions. In this context, factorization of a polynomial into polynomials of lower degree corresponds to the decomposition of a rational motion into "simpler" motions. One of the simplest non-trivial motions are rotations. They can be represented by linear polynomials. On the other hand, linear polynomials generically represent rotational motions. Hence, a motion described by a polynomial that admits a factorization into linear factors can be realized by a mechanism whose revolute joints correspond to the linear factors.

A suitable model for motions in the hyperbolic plane is provided by the non-commutative ring of split quaternions [2, Chapter 8]. In contrast to the (Hamiltonian) quaternions, the presence of zero divisors makes the factorization theory of polynomials over split quaternions more complex. Factorization of quadratic split quaternion polynomials has been investigated in [1] and [3]. Zeros of split quaternion polynomials of higher degree (which are closely related to linear factors, c.f. Lemma 2.9) are the topic of [7]. Based on the theory of motion factorization by means of quaternions $[8,14]$ and dual quaternions $[9,12]$ a characterization of factorizability for such polynomials has been found [15]. In order to compute factorizations, the algorithm of the Euclidean setup has been adapted $[11,15]$.

In this article, we consider rational motions in the hyperbolic plane, represented by polynomials over the split quaternions. We extend the results from the quadratic case [15] to polynomials of arbitrary degree. A main ingredient is a geometric interpretation of factorizability. We investigate the "geometry" of the factorization algorithm for generic cases and modify it such that it provably finds all factorizations into linear factors. Special cases include polynomials with infinitely many factorizations or no factorizations at all.

In case a split quaternion polynomial representing a rational motion has no factorization with linear factors we can adopt a "degree elevation technique" from the Euclidean setup [12]: multiplying with a suitable real polynomial does not change the underlying rational motion but allows the decomposition into linear factors. Hence, the initial motion can be decomposed into hyperbolic rotations. In contrast to the Euclidean case, our approach is rather based on geometric considerations than algebraic ones.

It is worth mentioning that the set of split quaternions is isomorphic to the fundamental algebra of real $2 \times 2$ matrices and all of our results can be directly transferred.

The further structure of this article is as follows: After a brief definition of split quaternions and hyperbolic motions in Sect. 2 we recall the state of art in factorization theory in Sect. 3.1. In Sect. 3.2 we introduce a new algorithm for factorization of split quaternion polynomials. In Sect. 3.3 we investigate the geometry of the algorithm and present a degree-elevation technique for dealing with non-factorizable polynomials as well. Finally, we apply some of 
our ideas to Euclidean motions in Sect. 3.5 and compute new factorization for dual quaternion polynomials.

\section{Preliminaries}

\subsection{Split Quaternions and Their Geometry}

The algebra of split quaternions $\mathbb{S}$ is defined as the set

$$
\left\{h=h_{0}+h_{1} \mathbf{i}+h_{2} \mathbf{j}+h_{3} \mathbf{k}: h_{0}, h_{1}, h_{2}, h_{3} \in \mathbb{R}\right\} .
$$

Addition of split quaternions is done component-wise and the non-commutative multiplication is obtained from the relations

$$
\mathbf{i}^{2}=-\mathbf{j}^{2}=-\mathbf{k}^{2}=-\mathbf{i j k}=-1 .
$$

The conjugate split quaternion of $h=h_{0}+h_{1} \mathbf{i}+h_{2} \mathbf{j}+h_{3} \mathbf{k}$ is defined by $h^{*}:=h_{0}-h_{1} \mathbf{i}-h_{2} \mathbf{j}-h_{3} \mathbf{k}$ and the expression

$$
h h^{*}=h^{*} h=h_{0}^{2}+h_{1}^{2}-h_{2}^{2}-h_{3}^{2} \in \mathbb{R}
$$

is sometimes called norm in the context of quaternions, even if it can attain negative values and lacks the square root. The center of $\mathbb{S}$ is precisely the field of real numbers $\mathbb{R}$, i.e. $h r=r h$ for all $r \in \mathbb{R}$. Later on we will also allow complex coefficients $h_{0}, h_{1}, h_{2}, h_{3} \in \mathbb{C}$ and multiply by complex numbers $z \in \mathbb{C}$ that commute with split quaternions as well. For the complex unit we will use the (non-boldface) symbol $\mathrm{i} \in \mathbb{C}$ with the property $\mathrm{i}^{2}=-1$. Conjugation of complex numbers is denoted by $\bar{z} \in \mathbb{C}$. Let $g \in \mathbb{S}$ be another split quaternion, then $(h g)^{*}=g^{*} h^{*}$ and $(h g)(h g)^{*}=h h^{*} g g^{*}$. The inverse of $h$ is $h^{-1}=\left(h h^{*}\right)^{-1} h^{*}$. It exists if and only if $h h^{*} \neq 0$. We call $\operatorname{Re}(h):=$ $\frac{1}{2}\left(h+h^{*}\right)=h_{0}$ the scalar part and $\operatorname{Im}(h):=\frac{1}{2}\left(h-h^{*}\right)=h_{1} \mathbf{i}+h_{2} \mathbf{j}+h_{3} \mathbf{k}$ the vector part of $h \in \mathbb{S}$. If $\operatorname{Re}(h)=0$ then $h$ is called vectorial. The scalar product and the cross product of $h$ and $g$ are defined by

$$
\langle h, g\rangle:=\frac{1}{2}\left(h g^{*}+g h^{*}\right) \quad \text { and } \quad h \times g:=\frac{1}{2}(h g-g h),
$$

respectively. Note that the cross product is not affected by the scalar parts, i.e. $h \times g=\operatorname{Im}(h) \times \operatorname{Im}(g)$. The real four-dimensional vector space of split quaternions together with the symmetric bilinear form

$$
\langle\cdot, \cdot\rangle: \mathbb{S} \times \mathbb{S} \rightarrow \mathbb{R}:(h, g) \mapsto\langle h, g\rangle
$$

is a pseudo-Euclidean space. The set

$$
\mathfrak{N}:=\{h \in \mathbb{S}:\langle h, h\rangle=0\}
$$

is called the null cone. It consists precisely of all zero divisors in $\mathbb{S}$. We will also consider the projective space $\mathbb{P}(\mathbb{S})$ over $\mathbb{S}$. An element of $\mathbb{P}(\mathbb{S})$ is denoted by $[h]$ where $h \in \mathbb{S} \backslash\{0\}$ is a non-zero split quaternion. Let $[g],[h] \in \mathbb{P}(\mathbb{S})$ be two elements. The projective span of $[h]$ and $[g]$ is denoted by

$$
[h] \vee[g]:=\left\{[\lambda h+\mu g] \in \mathbb{P}(\mathbb{S}):[\lambda, \mu]^{\top} \in \mathbb{P}(\mathbb{R})\right\} .
$$

It is a straight line in general and only a point if $[h]=[g]$, i.e. $h$ and $g$ are linearly dependent in the vector space $\mathbb{S}$. 
Definition 2.1. [15] The quadric $\mathcal{N}$ in $\mathbb{P}(\mathbb{S})$ represented by the symmetric bilinear form $\langle\cdot, \cdot\rangle$ is called the null quadric. Lines contained in $\mathcal{N}$ are called null lines or rulings of $\mathcal{N}$.

The null quadric $\mathcal{N}$ is of hyperbolic type because the signature of $\langle\cdot, \cdot\rangle$ is $(2,2)$. Moreover, $\mathcal{N}$ carries two families of lines. In the following we recall some basic results on null lines and the null quadric $\mathcal{N}$. For the proofs we refer to [13] and [15].

Theorem 2.2. [15] If [h] is a point of $\mathcal{N}$, the sets

$$
\mathcal{L}:=\left\{[g] \in \mathbb{P}(\mathbb{S}): g h^{*}=0\right\} \quad \text { and } \quad \mathcal{R}:=\left\{[g] \in \mathbb{P}(\mathbb{S}): h^{*} g=0\right\}
$$

are the two different rulings of $\mathcal{N}$ through $[h]$.

Definition 2.3. Consider a ruling $L \subset \mathcal{N}$. If $g h^{*}=0$ for all $[h],[g] \in L$, then $L$ is called a left ruling. If $h^{*} g=0$ for all $[h],[g] \in L$, then $L$ is called a right ruling.

Corollary 2.4. [15] Consider two points $[h] \in \mathcal{N}$ and $[g] \in \mathbb{P}(\mathbb{S})$.

- If $g$ is such that $g h \neq 0$ and $[g h] \neq[h]$, then $[h] \vee[g h]$ is a left ruling of $\mathcal{N}$.

- If $g$ is such that $h g \neq 0$ and $[h g] \neq[h]$, then $[h] \vee[h g]$ is a right ruling of $\mathcal{N}$.

Remark 2.5. For fixed $g \in \mathbb{S} \backslash \mathfrak{N}$ the maps $[x] \mapsto[g x]$ and $[x] \mapsto[x g]$ are the well-known Clifford left and right translations of non-Euclidean geometry (c.f. [15]). If $g \in \mathfrak{N} \backslash\{0\}$, these maps are still defined on $\mathbb{P}(\mathbb{S})$ minus the subspaces of left/right annihilators of $g$. We refer to them as singular Clifford left or right translations. Their image is the left/right ruling through $[g]$, respectively.

Theorem 2.6. [15] Consider two split quaternions $h=h_{0}+h_{1} \mathbf{i}+h_{2} \mathbf{j}+h_{3} \mathbf{k} \in$ $\mathbb{S} \backslash\{0\}$ and $g=g_{0}+g_{1} \mathbf{i}+g_{2} \mathbf{j}+g_{3} \mathbf{k} \in \mathbb{S} \backslash\{0\}$ such that $[h] \vee[g]$ is a left ruling of $\mathcal{N}$. There exists an affine two-plane consisting of all split quaternions $x \in \mathbb{S}$ solving the equation $g=x h$. It can be parameterized by $u+\lambda h^{*}+\mu \mathbf{i} h^{*}$, where $u=\left(g_{0}+g_{1} \mathbf{i}\right)\left(h_{0}+h_{1} \mathbf{i}\right)^{-1}$ and $\lambda, \mu \in \mathbb{R}$. (If $[h] \vee[g]$ is a right ruling, the same statement holds for $g=h x$ with $u+\lambda h^{*}+\mu h^{*} \mathbf{i}$ where $u=\left(h_{0}+h_{1} \mathbf{i}\right)^{-1}\left(g_{0}+g_{1} \mathbf{i}\right)$ and $\lambda, \mu \in \mathbb{R}$. It even holds true if $[h]$ and $[g]$ coincide and therefore do not span a straight line.)

\subsection{Hyperbolic Motions and Split Quaternion Polynomials}

In this section we recall the split quaternion model for hyperbolic motions of [2, Chapter 8] employing the terminology of [17-19]. Consider the threedimensional vector space

$$
\operatorname{Im}(\mathbb{S}):=\{h \in \mathbb{S}: \operatorname{Re}(h)=0\}
$$

of all vectorial split quaternions and the projective plane $H^{2}$ over $\operatorname{Im}(\mathbb{S})$. Points of $H^{2}$ are again denoted by $[h]$ where $h \in \operatorname{Im}(\mathbb{S}) \backslash\{0\}$. The null quadric $\mathcal{N}$ intersects $H^{2}$ in the absolute circle or null circle. 
A rotation in the hyperbolic plane $H^{2}$ is defined as the composition of two reflections. A reflection is a homology of $H^{2}$ in the sense of [4, Section 5.7] (a projective collineation, different from the identity, with a line of fixed points and a bundle of fixed lines) that preserves the absolute circle $\mathcal{N} \cap H^{2}$. The intersection point of all lines in the bundle of fixed lines is called the center of the reflection and the line of fixed points is called its axis. Center and axis are polar to each other with respect to the null circle. A rotation has a fix point as rotation center as well, the intersection point of the two reflection axes. In our setting it makes sense to consider reflections as special (idempotent) rotations because they are described by the same formula (1) in Theorem 2.7 below. Formally, this is accomplished by considering the identity as a reflection with unspecified center and axis.

The split quaternion approach allows to describe rotations in terms of split quaternion multiplication.

Theorem 2.7. [2, Chapter 8] Let $h \in \mathbb{S}$ be an invertible split quaternion. Then the map

$$
\varrho: H^{2} \rightarrow H^{2}:[x] \mapsto\left[h x h^{*}\right]
$$

is a rotation with rotation center $[\operatorname{Im}(h)] \in H^{2}$. It is a reflection if $\operatorname{Re}(h)=0$.

Rotations preserve distances with respect to the scalar product $\langle\cdot, \cdot\rangle$. Consider an invertible split quaternion $h \in \mathbb{S}$ and two points $[v],[w] \in H^{2}$ not contained in $\mathcal{N}$. The quadrance (squared distance) of $[v]$ and $[w]$ is defined by

$$
Q([v],[w]):=1-\frac{\langle v, w\rangle^{2}}{\langle v, v\rangle\langle w, w\rangle} .
$$

It is a concept of universal hyperbolic geometry in the sense of Wildberger [19]. In contrast to traditional hyperbolic geometry, the quadrance might be zero and even attain negative values. Because of

$$
\begin{aligned}
2\left\langle h v h^{*}, h w h^{*}\right\rangle & =h v h^{*} h w^{*} h^{*}+h w h^{*} h v^{*} h^{*} \\
& =\left(h h^{*}\right) h\left(v w^{*}+w v^{*}\right) h^{*}=2\left(h h^{*}\right)^{2}\langle v, w\rangle
\end{aligned}
$$

it is unchanged when displacing $[v]$ and $[w]$ via (1). Thus, rotations indeed preserve distances in $H^{2}$. For more details we refer to [2, Chapter 8].

The ring of polynomials in one indeterminate $t$ (where multiplication is defined by the convention that $t$ commutes with all coefficients) is denoted by $\mathbb{S}[t]$. This convention on multiplication is motivated by our kinematic interpretation of parametrizing motions in the hyperbolic plane via split quaternion polynomials. The parameter $t$ can be viewed as a real motion parameter. In the same spirit, we also define $t^{*}:=t$.

Consider a split quaternion polynomial $P=\sum_{\ell=0}^{n} p_{\ell} t^{\ell} \in \mathbb{S}[t]$ of degree $n \in \mathbb{N}$ with $p_{0}, \ldots, p_{n} \in \mathbb{S}$ and $p_{n} \neq 0$. The conjugate polynomial of $P$ is $\sum_{\ell=0}^{n} p_{\ell}^{*} t^{\ell}$ and its norm polynomial $P P^{*}=P^{*} P$ is a real polynomial. Right evaluation of $P$ at $h \in \mathbb{S}$ is defined by $P(h):=\sum_{\ell=0}^{n} p_{\ell} h^{\ell}$, i.e. the indeterminate $t$ is only substituted by $h$ after it is written to the right hand side of the coefficients in expanded form. This is important when evaluating 
a product of polynomials. Consider the two polynomials $t, \mathbf{i} \in \mathbb{S}[t]$. Their product evaluated at $\mathbf{j} \in \mathbb{S}$ is equal to $(t \mathbf{i})(\mathbf{j})=(\mathbf{i} t)(\mathbf{j})=\mathbf{i j}=\mathbf{k} \neq-\mathbf{k}=\mathbf{j i}$. A split quaternion $h \in \mathbb{S}$ is called a right zero of $P \in \mathbb{S}[t]$ if $P(h)=0$. Left evaluation and left zeros are defined in the same manner.

Definition 2.8. A split quaternion polynomial $P \in \mathbb{S}[t]$ of degree $n \geq 1$ admits a factorization into linear factors if there exist split quaternions $p$, $h_{1}, \ldots, h_{n} \in \mathbb{S}$ such that

$$
P=p\left(t-h_{1}\right) \ldots\left(t-h_{n}\right) .
$$

The rightmost factor $t-h_{n}$ in Definition 2.8 is linked to a right zero of $P$. We recall this result from [11].

Lemma 2.9. The split quaternion $h \in \mathbb{S}$ is a left/right zero of the polynomial $P \in \mathbb{S}[t]$ if and only if $t-h$ is a left/right factor of $P$.

In contrast to polynomials over the real or complex numbers, this statement does not hold for all linear factors of a split quaternion polynomial. Consider the polynomial $P=(t-\mathbf{j})(t-\mathbf{i})$. Evaluation of $P$ at $\mathbf{i}$ and $\mathbf{j}$ yields

$$
\begin{aligned}
& P(\mathbf{i})=(t-\mathbf{j})(t-\mathbf{i})(\mathbf{i})=\left(t^{2}-(\mathbf{i}+\mathbf{j}) t-\mathbf{k}\right)(\mathbf{i})=\mathbf{i}^{2}-\mathbf{i}^{2}-\mathbf{j i}-\mathbf{k}=0, \\
& P(\mathbf{j})=\mathbf{j}^{2}-\mathbf{i} \mathbf{j}-\mathbf{j}^{2}-\mathbf{k}=-2 \mathbf{k} \neq 0,
\end{aligned}
$$

respectively. Hence, $\mathbf{i}$ is a right zero of $P$ but $\mathbf{j}$ is not. However, $\mathbf{j}$ is a left zero of $P$.

Let $P \in \mathbb{S}[t]$ be a split quaternion polynomial with $P P^{*} \neq 0$. If we use $P$ instead of $h$ in Eq. (1) we obtain a one-parametric motion in $H^{2}$. The trajectories of a point $[x] \in H^{2}$ are given by

$$
[x] \mapsto\left[P(t) x P(t)^{*}\right] .
$$

They are rational curves parametrized by the parameter $t \in \mathbb{R}$. Since real numbers commute with split quaternions we have $P(t) x P(t)^{*}=\left(P x P^{*}\right)(t)$. If $P\left(t_{0}\right) x P\left(t_{0}\right)^{*}=0$ for some $t_{0} \in \mathbb{R}$ then the corresponding point $\left[P\left(t_{0}\right) x P\left(t_{0}\right)^{*}\right]$ is defined by continuity.

Note that we will later also consider parameter values $t \in \mathbb{C}$. Moreover, in order to get algebraically closed trajectories, we should allow the parameter value $t=\infty$ and define $P(\infty):=p$ (the leading coefficient of $P$ ).

In the following we will only consider a certain set of split quaternion polynomials, namely those which

1. have a norm polynomial different from zero,

2. are monic, i.e. their leading coefficient is equal to $1 \in \mathbb{R} \subset \mathbb{S}$ and

3. have no real or complex polynomial factors of positive degree.

The first and second assumptions are no loss of generality from a kinematic point of view. Polynomials with a vanishing norm polynomial do not describe proper motions because they map all point in $H^{2}$ onto $\mathcal{N}$. Let $P \in \mathbb{S}[t]$ be a polynomial such that $P P^{*} \neq 0$ and let $p \in \mathbb{S}$ be its leading coefficient. If $p$ is invertible, then $P$ admits a factorization into linear factors if and only if $p^{-1} P$ does. If $p$ is not invertible then we can apply a proper, bijective re-parametrization to $P$ and obtain a polynomial whose leading coefficient 
is invertible. The third item in our list is only a technical assumption for now and will be dropped later on. We call split quaternions without real or complex polynomial factors of positive degree reduced.

Assume that $P \in \mathbb{S}[t]$ is a reduced, monic split quaternion polynomial which admits a factorization into linear factors $P=\left(t-h_{1}\right) \ldots\left(t-h_{n}\right)$. By Theorem 2.7, each of the factors $t-h_{i} \in \mathbb{S}[t]$ represents a rotation with rotation center $\left[\operatorname{Im}\left(t-h_{i}\right)\right]=\left[\operatorname{Im}\left(h_{i}\right)\right]$. Therefore, $P$ represents the motion obtained from the composition of these rotations - the motion of an open kinematic chain of hyperbolic revolute joints [2, Chapter 8].

\section{Factorization Results}

\subsection{State of the Art}

In [9] the authors developed an algorithm to decompose generic "motion polynomials" into linear "motion polynomial factors". Motion polynomials are polynomials with coefficients in the non-commutative ring of dual quaternions whose coefficients satisfy a certain quadratic constraint, the Study condition, that ensures that the norm polynomial is real (c.f. Sect. 3.5). The factorization algorithm can be adapted for split quaternions and will succeed in "generic" cases. In Sect. 3.2 we will develop a modified version of this algorithm that provably succeeds whenever a factorization exists. In these cases, it can be used to compute all factorizations.

Let $P \in \mathbb{S}[t]$ be a reduced, monic split quaternion polynomial and let $N \in \mathbb{R}[t]$ be a quadratic factor of its norm polynomial $P P^{*} \in \mathbb{R}[t]$. Polynomial division of $P$ by $N$ yields unique polynomials $Q, R \in \mathbb{S}[t]$ such that $P=$ $Q N+R$ and $\operatorname{deg}(R)<\operatorname{deg}(N)=2$. Moreover, we have

$$
P P^{*}=(Q N+R)(Q N+R)^{*}=\left(Q Q^{*} N+Q R^{*}+R Q^{*}\right) N+R R^{*}
$$

and $N$ divides $R R^{*}$. If the leading coefficient of $R=r_{1} t+r_{0} \in \mathbb{S}[t]$ is invertible, then $h:=-r_{1}^{-1} r_{0} \in \mathbb{S}$ is the unique right zero of $R$. Since $N$ divides $R R^{*}=R^{*} R, h$ is also a right zero of $N$ and hence also of $P=Q N+R$. By Lemma $2.9, t-h \in \mathbb{S}[t]$ is a right factor of $P$ which therefore can be written as $P=P^{\prime}(t-h)$. An inductive application of the procedure above yields a (generic) factorization algorithm [11].

Remark 3.1. A different choice of the quadratic factor $N$ yields a different right factor $t-h$. Therefore, the number of factorizations of $P$ depends on the number of distinct real quadratic factors of $P P^{*}$. The factorization algorithm can also be adapted to compute left factors: The unique left zero $g:=-r_{0} r_{1}^{-1}$ of $R$ yields a left factor $t-g \in \mathbb{S}[t]$ of $P$.

A pseudocode listing is provided in Algorithm 1. For the sake of brevity, it uses the following notations: Let $G \in \mathbb{S}$ be a polynomial with invertible leading coefficient. Due to non-commutativity of split quaternion multiplication we have to distinguish between left and right polynomial division. The unique polynomials $Q, R \in \mathbb{S}[t]$ such that $P=G Q+R(P=Q G+R)$ are called the right (left) quotient and right (left) remainder of $P$ and $G$. They are denoted by $Q=\operatorname{rquo}(P, G)$ and $R=\operatorname{rrem}(P, G)(\operatorname{lquo}(P, G)$ and $\operatorname{lrem}(P, G))$. 
If $G$ is a real polynomial, then the left/right quotient and the left/right remainder coincide, respectively. We denote them by quo $(P, G)$ and $\operatorname{rem}(P, G)$. Moreover, we use the symbol " $\oplus$ " to denote non-commutative concatenation of tuples, i.e. $L_{1} \oplus L_{2}$ is the tuple which starts with the elements of the tuple $L_{1}$ and ends with the elements of the tuple $L_{2}$.

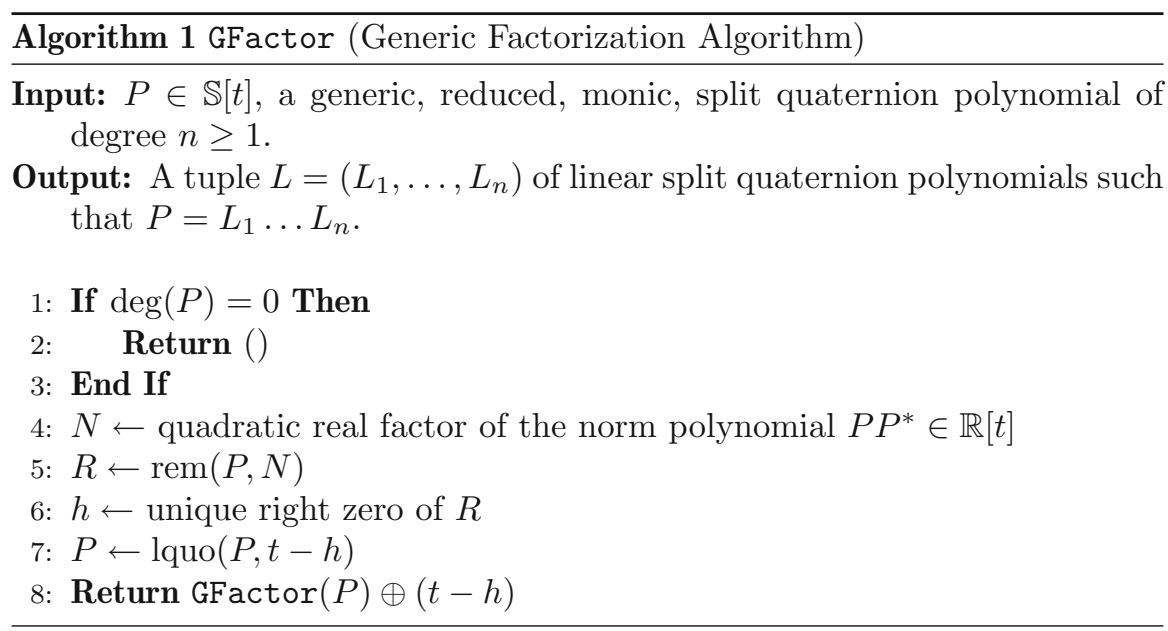

The term "generic" in Algorithm 1 refers to $R$ having a unique right zero in each iteration. This is equivalent to the condition $R R^{*} \neq 0$ because $N$ divides $R R^{*}$ and therefore $\operatorname{deg}\left(R R^{*}\right)=2$ or $R R^{*}=0$. The latter one implies that the leading coefficient of $R$ is not invertible and $h$ in Algorithm 1 is not well-defined. In fact, we have:

Lemma 3.2. [13] Let $R=r_{1} t+r_{0} \in \mathbb{S}[t]$ be a split quaternion polynomial of degree one with linearly independent coefficients $r_{0}, r_{1} \in \mathbb{S}$. Then the set $\{[R(t)]: t \in \mathbb{R} \cup\{\infty\}\}$ is the straight line spanned by $\left[r_{0}\right]$ and $\left[r_{1}\right]$. It is a null line if and only if $R R^{*}=0$.

We often will say that $R$ "parametrizes" the straight line spanned by $\left[r_{0}\right]$ and $\left[r_{1}\right]$. Theorem 2.6 and Lemma 3.2 imply that a polynomial $R$ as in Lemma 3.2 and with $R R^{*}=0$ has an affine two-parametric set of right or left zeros, respectively.

Example 3.3. Consider the polynomial $P_{1}=t^{2}-(\mathbf{i}+\mathbf{j}) t-\mathbf{k}$. The norm polynomial $P_{1} P_{1}{ }^{*}=t^{4}-1$ has the two quadratic real factors $N_{1}=t^{2}+1$ and $N_{2}=t^{2}-1$. Their respective remainder polynomials $R_{1}=\operatorname{rem}\left(P_{1}, N_{1}\right)=$ $-(\mathbf{i}+\mathbf{j}) t-1-\mathbf{k}$ and $R_{2}=\operatorname{rem}\left(P_{1}, N_{2}\right)=-(\mathbf{i}+\mathbf{j}) t+1-\mathbf{k}$ parametrize null lines because $R_{1} R_{1}^{*}=R_{2} R_{2}^{*}=0$. Moreover, $R_{1}$ parametrizes a right ruling of $\mathcal{N}$ and $R_{2}$ parametrizes a left ruling because

$$
-(\mathbf{i}+\mathbf{j})^{*}(-1-\mathbf{k})=0=-(\mathbf{i}+\mathbf{j})(1-\mathbf{k})^{*} .
$$

Hence, $R_{1}$ has infinitely many right zeros and $R_{2}$ has infinitely many left zeros. 
The statements on left/right zeros are no longer true if the coefficients of $R$ are linearly dependent. Recall that in this case $R$ "parameterizes" a single point on $\mathcal{N}$.

\subsection{Factorization of Non-Generic Polynomials}

In this section we generalize the results from Sect. 3.1 and investigate methods to compute factorizations into linear polynomials of reduced, monic split quaternion polynomials, including non-generic polynomials. In addition, we provide an algorithm for computing such factorizations. To do so, we recall a result on the split quaternion zeros of a real polynomial.

Lemma 3.4. $[10,15]$ Let $P=t^{2}+b t+c \in \mathbb{R}[t]$ be a real polynomial. The set of zeros of $P$ in $\mathbb{S} \backslash \mathbb{R}$ is given by

$$
\left\{\frac{1}{2}\left(-b+h_{1} \mathbf{i}+h_{2} \mathbf{j}+h_{3} \mathbf{k}\right): h_{1}^{2}-h_{2}^{2}-h_{3}^{2}=4 c-b^{2}\right\} .
$$

In particular, the set of split quaternion zeros of a quadratic real polynomial is two-parametric.

Remark 3.5. By Lemma 2.9, real polynomials of degree greater than one admit infinitely many factorizations over $\mathbb{S}$. Depending on the sign of $4 c-b^{2}$ $(<0,=0$ or $>0)$ the set of zeros in Lemma 3.4 describes a hyperboloid of one sheet, a quadratic cone or a hyperboloid of two sheets, respectively, in the three-dimensional affine space $\left\{h \in \mathbb{S}: \operatorname{Re}(h)=-\frac{b}{2}\right\}$ (Fig. 1). If $b=0$, this three-space equals the vector space $\operatorname{Im}(\mathbb{S})$.

Lemma 3.6. Consider a reduced, monic split quaternion polynomial $P \in \mathbb{S}[t]$ and let $N \in \mathbb{R}[t]$ be a quadratic factor of its norm polynomial $P P^{*}$. If $R:=$ $\operatorname{rem}(P, N)$ parametrizes a right ruling of $\mathcal{N}$, then there exists a unique split quaternion $h \in \mathbb{S}$ such that $t-h \in \mathbb{S}[t]$ is a right factor of $P$ and $N=$ $(t-h)\left(t-h^{*}\right)$. (The same statement holds for $R$ parametrizing a left ruling yielding a left factor.)

Proof. Let $R=r_{1} t+r_{0}$ parametrize the right ruling $\left[r_{0}\right] \vee\left[r_{1}\right] \subset \mathcal{N}$ (c.f. Lemma 3.2) and define $Q:=\operatorname{quo}(P, N)$. Because of $P=Q N+R$, the sought common right zero $h$ of $P$ and $N$ must also be a common right zero of $N$ and $R$, and vice versa. We will show that there exists a unique split quaternion $h$ with this property. The proof proceeds with geometric descriptions of the zero sets of $N$ and $R$ in the affine space of split quaternions. Their unique intersection point is the common zero $h$.

A split quaternion is a zero of $R$ if and only if it solves the equation $-r_{0}=r_{1} x$. According to Theorem 2.6, there exists an affine two-parametric plane of right zeros of $R$, let's call it $W \subset \mathbb{S}$. It is parallel to the twodimensional vector space $V:=\left\{\lambda r_{1}^{*}+\mu r_{1}^{*} \mathbf{i}: \lambda, \mu \in \mathbb{R}\right\}$ which parametrizes the solution set of the homogeneous equation $r_{1} x=0$. We compute

$$
\begin{aligned}
& \left(\lambda r_{1}^{*}+\mu r_{1}^{*} \mathbf{i}\right)\left(\lambda r_{1}^{*}+\mu r_{1}^{*} \mathbf{i}\right)^{*}=\left(\lambda r_{1}^{*}+\mu r_{1}^{*} \mathbf{i}\right)\left(\lambda r_{1}-\mu \mathbf{i} r_{1}\right) \\
& \quad=\lambda^{2} r_{1}^{*} r_{1}-\lambda \mu r_{1}^{*} \mathbf{i} r_{1}+\lambda \mu r_{1}^{*} \mathbf{i} r_{1}+\mu^{2} r_{1}^{*} r_{1}=\left(\lambda^{2}+\mu^{2}\right) r_{1}^{*} r_{1}=0,
\end{aligned}
$$

implying that $V$ is contained in the null cone $\mathfrak{N}$. 
Write $N=t^{2}+b t+c$ with $b, c \in \mathbb{R}$. By applying the parameter transformation $t \mapsto t-\frac{b}{2}$ to $P$ we can assume without loss of generality that $b=0$. Set $L:=W \cap \operatorname{Im}(\mathbb{S})$ and define $Z$ as the set of split quaternion zeros of $N$ by Lemma 3.4 .

The affine two-plane $W$ can not be contained in $\operatorname{Im}(\mathbb{S})$ because then $V$ is also contained in $\operatorname{Im}(\mathbb{S})$ and $\operatorname{Re}\left(\lambda r_{1}^{*}+\mu r_{1}^{*} \mathbf{i}\right)=0$ for all $\lambda, \mu \in \mathbb{R}$ which, together with $r_{1} r_{1}^{*}=0$, yields $r_{1}=0$. This is a contradiction to the assumption that $\left[r_{0}\right] \vee\left[r_{1}\right]$ spans a ruling of $\mathcal{N}$. By simply counting dimensions, we infer that $L$ is an affine line in the three-dimensional vector space $\operatorname{Im}(\mathbb{S})$. Since $W$ is parallel to $V$ and $V \subset \mathfrak{N}$, the line $L$ is parallel to a ruling of the affine cone $\mathfrak{N} \cap \operatorname{Im}(\mathbb{S})$. The later one is the asymptotic cone of $Z$ in $\operatorname{Im}(\mathbb{S})$. Therefore, the line $L$ intersects $Z$ in precisely one point if $L$ is not contained in a tangent-plane of $\mathfrak{N} \cap \operatorname{Im}(\mathbb{S})$ (Fig. 1). We will see below that this is indeed the case. Hence, $L$ and $Z$ indeed intersect in a point $h \in \mathbb{S}$ which is the unique common right zero of $N$ and $R$ and yields the right factor $t-h \in \mathbb{S}[t]$ of $P$ by Lemma 2.9 .

Thus, the proof is finished if we can show that $L$ is not contained in a tangent plane of the cone $\mathfrak{N} \cap \operatorname{Im}(\mathbb{S})$. Assume the opposite, i.e. let $L$ be contained in the tangent plane of an element $0 \neq p \in \mathfrak{N} \cap \operatorname{Im}(\mathbb{S})$. Then $L$ is parallel to the ruling through $p$, let's call it $L_{p}$. The Euclidean common normal of $L$ and $L_{p}$ through $0 \in \operatorname{Im}(\mathbb{S})$ is spanned by $p \times \mathbf{i} p \mathbf{i}$, where $\mathbf{i} p \mathbf{i}$ is the reflection of $p$ in the plane spanned by $\mathbf{j}$ and $\mathbf{k}$ and " $\times$ " refers to the Euclidean cross product in the three-dimensional vector space $\operatorname{Im}(\mathbb{S})$. Hence, there exists an $\alpha \in \mathbb{R}$ such that $\alpha(p \times \mathbf{i} p \mathbf{i}) \in L$. Therefore $\alpha(p \times \mathbf{i} p \mathbf{i})$ is a zero of $R$, i.e. $r_{0}=-r_{1} \alpha(p \times \mathbf{i} p \mathbf{i})$. Parallelity of $L$ and $L_{p}$ yields $p \in V$ and there exist $\beta, \gamma \in \mathbb{R}$ such that $p=\beta r_{1}^{*}+\gamma r_{1}^{*} \mathbf{i}$. Inserting this identity into $r_{0}=-r_{1} \alpha(p \times \mathbf{i} p \mathbf{i})$ and using the properties $r_{1} r_{1}^{*}=0$ and $p \in \operatorname{Im}(\mathbb{S})$ imply linear dependency of $r_{1}$ and $r_{0}$. This is a contradiction to $\left[r_{0}\right] \vee\left[r_{1}\right]$ spanning a ruling of $\mathcal{N}$.

Remark 3.7. Computing the unique zero $h$ of $P$ in Lemma 3.6 is easy. We have to solve the quadratic system resulting from $R(h)=N(h)=0$. Plugging the solution of the linear sub-system $R(h)=0$ into $N(h)=0$ will result in a linear system with exactly one solution. This not only works if $R R^{*}=0$ but also in generic cases.

Example 3.8. Consider the polynomial $P_{1}=t^{2}-(\mathbf{i}+\mathbf{j}) t-\mathbf{k}$ from Example 3.3 and the factors $N_{1}=t^{2}+1, N_{2}=t^{2}-1$ of $P_{1} P_{1}^{*}$. Among the infinitely many right zeros of $R_{1}=\operatorname{rem}\left(P_{1}, N_{1}\right)=-(\mathbf{i}+\mathbf{j}) t-1-\mathbf{k}$, there is only one zero of $N_{1}$, namely $\mathbf{i} \in \mathbb{S}$. Right division of $P_{1}$ by $t-\mathbf{i}$ yields the factorization $P_{1}=(t-\mathbf{j})(t-\mathbf{i})$. The same result can be obtained by computing $\mathbf{j} \in \mathbb{S}$ as the common left zero of $R_{2}=\operatorname{rem}\left(P_{1}, N_{2}\right)$ and $N_{2}$.

The only case we did not discuss yet is when the coefficients of $R$ are linearly dependent. We can write $R=\lambda r t+\mu r=(\lambda t+\mu) r \in \mathbb{S}[t]$ for some $\lambda, \mu \in \mathbb{R}$ and $r \in \mathbb{S}$. Assume that $R R^{*} \neq 0$, then $r r^{*} \neq 0$. Since $N$ divides $R R^{*}=(\lambda t+\mu)^{2} r r^{*}$ we infer that $N$ and $R$ have the common real zero $-\frac{\mu}{\lambda}$. But then $P$ has a real zero which is a contradiction to our assumption of $P$ 

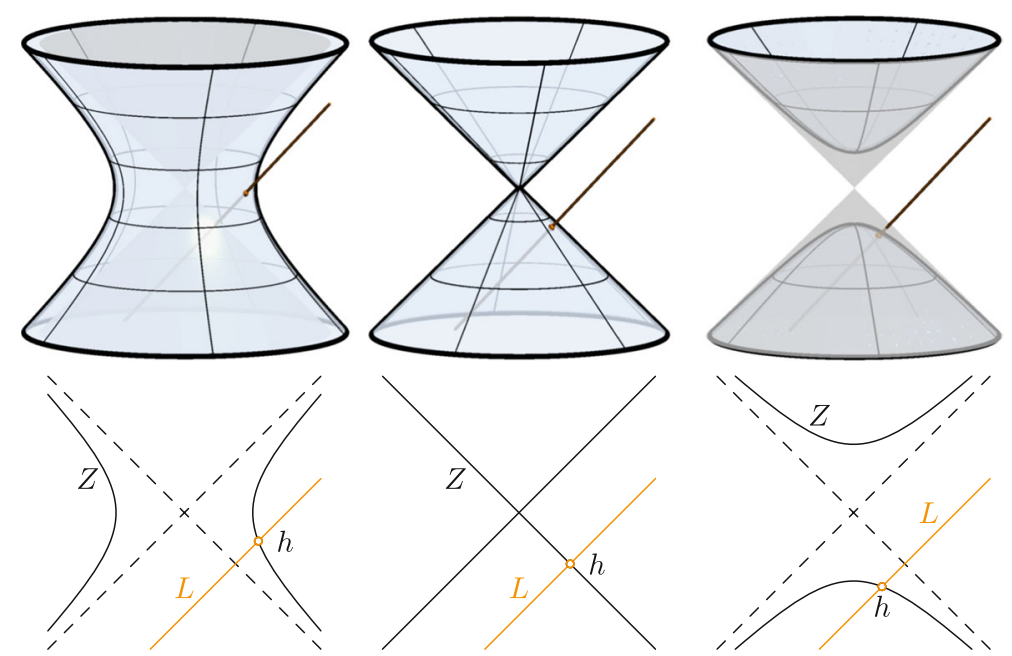

Figure 1. Intersection of the line $L$ and the set of zeros of $N$ from the proof of Lemma 3.6 for $N=t^{2}-1, N=t^{2}$ and $N=t^{2}+1$, respectively

being reduced. Hence, the case that $R$ has linearly dependent coefficient can only appear if $R R^{*}=0$. Then $R$ does not parametrize a null line but only a point $[r] \in \mathcal{N}$.

Example 3.9. Consider the polynomial $P_{2}=t^{2}+\mathbf{k}$. Its norm polynomial factors as $P_{2} P_{2}{ }^{*}=N_{1} N_{2}=\left(t^{2}+1\right)\left(t^{2}-1\right)$. The respective remainder polynomials $R_{1}=\operatorname{rem}\left(P_{2}, N_{1}\right)=-1+\mathbf{k}$ and $R_{2}=\operatorname{rem}\left(P_{2}, N_{2}\right)=1+\mathbf{k}$ do not have a zero in $\mathbb{S}$. A straightforward computation shows that $P_{2}$ does neither have a right zero nor a left zero and thus does not admit a factorization into linear factors.

The next lemma shows that only remainder polynomials of a certain type can occur in the presence of linear right factors.

Lemma 3.10. Consider a reduced, monic split quaternion polynomial $P \in$ $\mathbb{S}[t]$. Let $t-h \in \mathbb{S}[t]$ be a left/right factor of $P$ and let $N=(t-h)\left(t-h^{*}\right) \in \mathbb{R}[t]$. Then the remainder polynomial $R=\operatorname{rem}(P, N)$ parametrizes either a nonnull line or a left/right ruling of $\mathcal{N}$.

Proof. We only prove the statement for right factors. If $R R^{*} \neq 0$, the coefficients of $R$ are linearly independent by the considerations following Example 3.8. Moreover, $R$ parametrizes a non-null line by Lemma 3.2.

Next, we assume $R R^{*}=0$. The split quaternion $h \in \mathbb{S}$ is a right zero of $N$ as well as of $P$ by Lemma 2.9. Define $Q=q u o(P, N)$, then $h$ is a right zero of $R=P-Q N=r_{1} t+r_{0}$, i. e. $-r_{0}=r_{1} h$. Provided $r_{0}$ and $r_{1}$ are linearly independent, the points $\left[r_{0}\right]$ and $\left[r_{1}\right]$ are well defined and, by Corollary 2.4, span a right ruling. We proceed by showing linear independence of $r_{0}$ and $r_{1}$. Assume, on the contrary, that $R=\lambda r t+\mu r \in \mathbb{S}[t]$ for some $\lambda, \mu \in \mathbb{R}$ and 
$r \in \mathbb{S} \backslash\{0\}$. We have $0=R(h)=(\lambda r h+\mu r)=r(\lambda h+\mu)$ and $\lambda h+\mu$ is a zero divisor. If $\lambda=0$ this implies that $\mu=0$ and moreover $R=0$. Consequently, $P=Q N$ is not reduced, which is a contradiction to our assumption. Hence, $\lambda \neq 0$ and $h+\frac{\mu}{\lambda}$ is a zero divisor, i. e. $0=\left(h+\frac{\mu}{\lambda}\right)\left(h+\frac{\mu}{\lambda}\right)^{*}=\left(h+\frac{\mu}{\lambda}\right)\left(h^{*}+\frac{\mu}{\lambda}\right)=$ $N\left(-\frac{\mu}{\lambda}\right)$. Again, we obtain a contradiction since we infer that $P=Q N+R$ has the real zero $-\frac{\mu}{\lambda}$ and is not reduced.

Assume that $P$ admits a factorization into linear factors $P=(t-$ $\left.h_{1}\right) \ldots\left(t-h_{n}\right)$. The rightmost factor $t-h_{n}$ can be computed by using the real polynomial $N=\left(t-h_{n}\right)\left(t-h_{n}^{*}\right)$ and $R=\operatorname{rem}(P, N)$. By Lemma 3.10, $R$ parametrizes either a non-null line and we run one iteration of Algorithm 1 , or $R$ parametrizes a right ruling of $\mathcal{N}$ and we compute $t-h_{n}$ as explained in Remark 3.7. The complete factorization can be obtained by an iterative procedure using the real polynomials $\left(t-h_{n-1}\right)\left(t-h_{n-1}^{*}\right), \ldots,\left(t-h_{1}\right)\left(t-h_{1}^{*}\right) \in$ $\mathbb{R}[t]$.

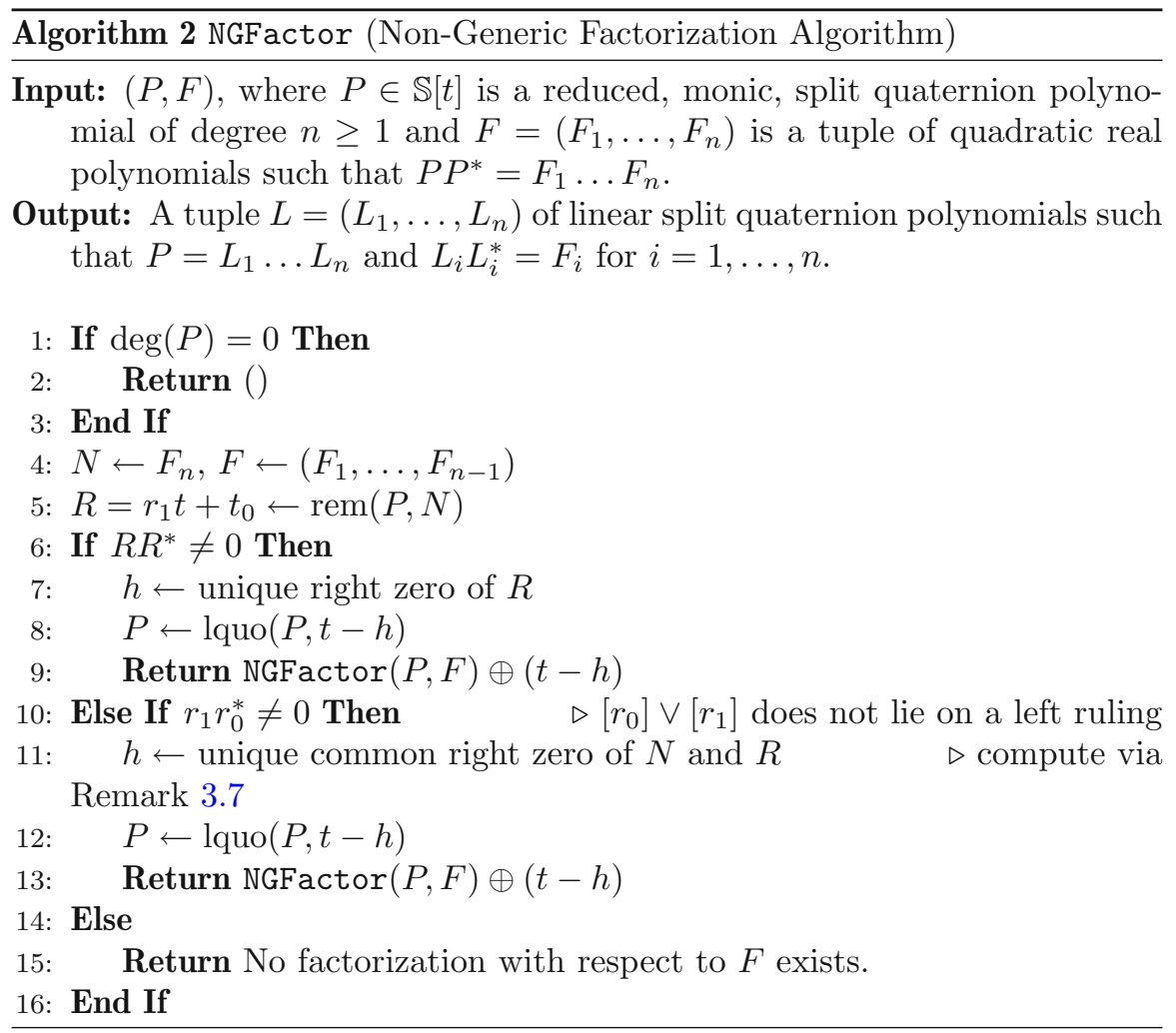

These considerations are transformed into Algorithm 2 for computing factorizations also in non-generic cases. It attempts to recursively compute a factorization from a given sequence $\left(F_{1}, \ldots, F_{n}\right)$ of quadratic real factors of the norm polynomial $P P^{*}$. In doing so, it only tries to find right factors. If this fails, it stops and returns a factorization of a right factor. The next theorem justifies the restriction to right factors only. 
Theorem 3.11. A reduced, monic split quaternion polynomial $P \in \mathbb{S}[t]$ of degree $n \geq 1$ admits a factorization into linear factors if an only if Algorithm 2 finds a factorization for some tuple $F=\left(F_{1}, \ldots, F_{n}\right)$ consisting of quadratic real polynomials such that $P P^{*}=F_{1} \ldots F_{n}$.

Proof. We have already argued the correctness of Algorithm 2 so that a factorization exists, if the algorithm does not stop prematurely. On the other hand, the considerations after the proof of Lemma 3.10 show that Algorithm 2 will recursively find the linear factors of the factorizations $P=$ $\left(t-h_{1}\right) \ldots\left(t-h_{n}\right)$ for the input $F=\left(F_{1}, \ldots, F_{n}\right)$ with $F_{i}=\left(t-h_{i}\right)\left(t-h_{i}^{*}\right), i \in$ $\{1, \ldots, n\}$.

Remark 3.12. Algorithm 2 relies on the possibility to compute factorizations of the real norm polynomial $P P^{*}$. In general, this requires numerical approximation which is not naturally incorporated into our otherwise symbolic algorithm. Nonetheless, it should be considered. We believe that this should be possible in a satisfactory way but refrain from pursuing this question in this article.

Example 3.13. Once more, we consider the polynomial $P_{1}=t^{2}-(\mathbf{i}+\mathbf{j}) t-\mathbf{k}$ from Example 3.3. As we have seen in Example 3.8, Algorithm 2 computes a factorization for the input $\left(P,\left(N_{2}, N_{1}\right)\right)$. It will fail for the input $\left(P,\left(N_{1}, N_{2}\right)\right)$ because $R_{2}=-(\mathbf{i}+\mathbf{j}) t-1-\mathbf{k}$ parametrizes a left ruling of $\mathcal{N}$ and thus has no right zeros. Of course, one could device a left version of Algorithm 2 as well.

Example 3.14. For every integer $n>1$, there exist polynomials of degree $n$ without linear factors. One example is $t^{n}+\mathbf{i}+\mathbf{k}$. Its norm polynomial equals $t^{2 n}$. Thus, the only quadratic factor to use in Algorithm 2 is $t^{2}$. We have $\operatorname{rem}\left(t^{n}+\mathbf{i}+\mathbf{k}, t^{2}\right)=\mathbf{i}+\mathbf{k}-$ a constant polynomial which does not have any zero.

\subsection{Geometry of the Factorization Algorithm}

In this section we will investigate the "geometry" of Algorithm 2 in order to clarify the cause of non-factorizability. By Theorem 3.11, a reduced, monic split quaternion polynomial $P$ does not admit a factorization into linear factors if and only if the algorithm fails for any order of the quadratic real factors $F_{i}$. Let $P=P^{\prime}\left(t-h_{m}\right) \ldots\left(t-h_{n}\right)$ for some $m \in\{1, \ldots, n\}$ and a polynomial $P^{\prime} \in \mathbb{S}[t]$ that does not have a linear right factor. Moreover, let $N \in \mathbb{R}[t]$ be a quadratic factor of $P^{\prime} P^{\prime *}$. According to Lemma 3.6 and the considerations in Sect. 3.1, $R=\operatorname{rem}\left(P^{\prime}, N\right)$ can not have a right zero and must either parametrize a left ruling of $\mathcal{N}$ or a point $[r] \in \mathcal{N}$, i.e. $[R(t)]=[r] \in \mathcal{N}$ for all $t \in \mathbb{R} \cup\{\infty\}$. Let $t_{1}, t_{2} \in \mathbb{C}$ be such that $N=\left(t-t_{1}\right)\left(t-t_{2}\right)$. For the time being we assume that $t_{1}, t_{2} \in \mathbb{R}$. For $i=1,2$ we have

$$
P\left(t_{i}\right)=P^{\prime}\left(t_{i}\right)\left(t_{i}-h_{m}\right) \ldots\left(t_{i}-h_{n}\right)
$$

and the point $\left[P\left(t_{i}\right)\right] \in \mathcal{N}$ is the image of $\left[P^{\prime}\left(t_{i}\right)\right] \in \mathcal{N}$ under the Clifford right translation $[x] \mapsto\left[x\left(t_{i}-h_{m}\right) \ldots\left(t_{i}-h_{n}\right)\right]$, c. f. Corollary 2.4 and Remark 2.5. The point $\left[P^{\prime}\left(t_{i}\right)\right]$ is contained in the pre-image of $\left[P\left(t_{i}\right)\right]$ if the Clifford right 
translation is singular and equals $\left[P\left(t_{i}\right)\right]$ otherwise. The failure of Algorithm 2 is equivalent to the line $\left[P\left(t_{1}\right)\right] \vee\left[P\left(t_{2}\right)\right]$ being contained in the pre-image of $\left[P^{\prime}\left(t_{1}\right)\right] \vee\left[P^{\prime}\left(t_{2}\right)\right]$, which is either a left ruling of $\mathcal{N}$ or degenerates to a single point $[r] \in \mathcal{N}$.

Example 3.15. Consider the polynomial $P_{3}=t^{3}-\mathbf{i} t^{2}+\mathbf{k} t-\mathbf{j}$. The norm polynomial is $P_{3} P_{3}{ }^{*}=\left(t^{2}+1\right)^{2}\left(t^{2}-1\right)$ and we can run Algorithm 2 with the following triples:

$$
\left(t^{2}-1, t^{2}+1, t^{2}+1\right),\left(t^{2}+1, t^{2}-1, t^{2}+1\right),\left(t^{2}+1, t^{2}+1, t^{2}-1\right) .
$$

The latter one yields the remainder polynomial $R_{1}=\operatorname{rem}\left(P_{3}, t^{2}-1\right)=(1+$ $\mathbf{k}) t-\mathbf{i}-\mathbf{j}$ and the right factor $t-j$ and $P_{3}^{\prime}=\operatorname{lquo}\left(P_{3}, t-\mathbf{j}\right)=t^{2}+(\mathbf{j}-\mathbf{i}) t+1$ in the first iteration. In the second iteration we obtain the remainder polynomial $\operatorname{rem}\left(P_{3}^{\prime}, t^{2}+1\right)=(\mathbf{j}-\mathbf{i}) t$ which parametrizes only the point $[\mathbf{j}-\mathbf{i}] \in \mathcal{N}$ and the algorithm stops. The polynomial $R_{1}$ parametrizes a right ruling of $\mathcal{N}$ which is contained in the pre-image of $[\mathbf{j}-\mathbf{i}]$.

Both of the other two triples yield the remainder polynomial $R_{2}=$ $\operatorname{rem}\left(P_{3}, t^{2}+1\right)=(\mathbf{k}-1) t+\mathbf{i}-\mathbf{j}$ and the right factor $t-\mathbf{i}$ and $P_{3}^{\prime}=\operatorname{lquo}\left(P_{3}, t-\right.$ $\mathbf{i})=t^{2}+\mathbf{k}$ in the first iteration. We have already seen in Example 3.9 that $t^{2}+\mathbf{k}$ does not admit a factorization because both remainder polynomials $\operatorname{rem}\left(P_{3}^{\prime}, t^{2}-1\right)=1+\mathbf{k}$ and $\operatorname{rem}\left(P_{3}^{\prime}, t^{2}+1\right)=-1+\mathbf{k}$ of the second iteration parametrize only a point on $\mathcal{N}$. The right ruling of $\mathcal{N}$ parametrized by $R_{2}$ is contained in the pre-image of $[-1+\mathbf{k}]$ and $[1+\mathbf{k}]$, respectively, with respect to the according Clifford right translations.

Hence, Algorithm 2 fails for all triples of quadratic real factors and $P_{3}$ does not admit a factorization.

If $t_{1}=\bar{t}_{2} \in \mathbb{C} \backslash \mathbb{R}$, then the coefficients of $R\left(t_{1}\right)$ and $R\left(t_{2}\right)$ might be complex numbers. However, all definitions and results from Sect. 2.1 can be translated to the ring of split quaternions with coefficients in $\mathbb{C}$. This has been done in [16] with the Hamiltonian quaternions. Considering complex numbers as coefficients they are isomorphic to the (complex) split quaternions. In addition, we extend the definition of the null cone $\mathfrak{N}$ as well as the null quadric $\mathcal{N}$ by also considering the split quaternions with complex coefficients fulfilling their defining equations, respectively. Note that the affine plane in Theorem 2.6 extends to an affine plane of complex dimension two in this setup.

If a split quaternion polynomial admits a factorization into linear factors then it must have a linear left/right factor and therefore a left/right zero by Lemma 2.9. Both potential remainder polynomials of $P_{3}$ in Example 3.15 $\operatorname{rem}\left(P_{3}, t^{2}-1\right)=(1+\mathbf{k}) t-\mathbf{i}-\mathbf{j}$ and $\operatorname{rem}\left(P_{3}, t^{2}+1\right)=(\mathbf{k}-1) t+\mathbf{i}-\mathbf{j}$ parametrize right rulings of $\mathcal{N}$. Hence, $P_{3}$ has no left zeros and we could state that is does not admit a factorization without the necessity to run Algorithm 2 with all triples of quadratic real factors of $P_{3} P_{3}{ }^{*}$. This result can be easily generalized.

Theorem 3.16. Consider a reduced, monic split quaternion polynomial $P \in$ $\mathbb{S}[t]$. If all sets $\{[R(t)] \subset \mathbb{P}(\mathbb{S}): t \in \mathbb{R} \cup\{\infty\}\}$ parametrized by its remainder 
polynomials after division by quadratic real factors of $P P^{*}$ belong to only on family of rulings of $\mathcal{N}$, then $P$ does not admit a factorization into linear factors.

Proof. $P$ admits a factorization into linear factors if and only if $P^{*}$ does. Moreover, conjugation of split quaternions interchanges the two families of rulings of $\mathcal{N}$. Hence, we can, without loss of generality, assume that all remainder polynomials of $P$ parametrize (subsets of) left rulings of $\mathcal{N}$. Then $P$ has no linear right factor by Lemma 3.10 and therefore does not admit a factorization into linear factors.

\subsection{Factorizing Hyperbolic Motions}

Since there exist split quaternions which do not admit a factorization into linear factors we can not unconditionally use the standard factorization theory in order to factor motions described by such polynomials. However, the projective nature of our kinematic model allows to multiply a split quaternion polynomial $P$ by a real polynomial $T \in \mathbb{R}[t]$. The product $T P$ describes the same motion as $P$ and may admit a factorization into linear factors. In this case, we can still decompose the initial motion into rotations even though $P$ does not admit a factorization. In the following we will show that such a polynomial $T$ always exists.

Theorem 3.17. Consider a reduced, monic split quaternion polynomial $P \in$ $\mathbb{S}[t]$. There exists a real polynomial $T \in \mathbb{R}[t]$ such that $T P$ admits a factorization into linear factors.

Proof. If $P$ already admits a factorization we can choose $T=1$. Assume that $P$ does not admit a factorization into linear factors. Without loss of generality we can assume that $P$ has no right factors. Otherwise we run Algorithm 2 with a suitable tuple of quadratic real factors of $P P^{*}$ and continue with the input polynomial in the iteration where the algorithm stops. Let $N=$ $\left(t-t_{1}\right)\left(t-t_{2}\right) \in \mathbb{R}[t]$ be a quadratic, real factor of $P P^{*}$ with $t_{1}, t_{2} \in \mathbb{C}$. By the considerations at the beginning of Sect. 3.3, $\left[P\left(t_{1}\right)\right] \vee\left[P\left(t_{2}\right)\right]$ is either a left ruling of $\mathcal{N}$ or $\left[P\left(t_{1}\right)\right]=\left[P\left(t_{2}\right)\right] \in \mathcal{N}$.

Consider $H=t-h \in \mathbb{S}[t]$ and denote by $z, \bar{z} \in \mathbb{C} \backslash \mathbb{R}$ the conjugate complex zeros of $H H^{*}=(t-z)(t-\bar{z}) \in \mathbb{R}[t]$. We will later argue that we can select $H$ such that

1. $P P^{*}(z) \neq 0 \neq P P^{*}(\bar{z})$,

2. $\left[H\left(t_{1}\right) P\left(t_{1}\right)\right] \neq\left[H\left(t_{2}\right) P\left(t_{2}\right)\right]$,

3. $[H(z) P(z)] \neq[H(\bar{z}) P(\bar{z})]$ and $[H(z) P(z)] \vee[H(\bar{z}) P(\bar{z})]$ is no left ruling of $\mathcal{N}$.

By Remark 2.5 and Item 2, the points $\left[H\left(t_{1}\right) P\left(t_{1}\right)\right]$ and $\left[H\left(t_{2}\right) P\left(t_{2}\right)\right]$ span the left ruling through $\left[P_{1}\right]$ and $\left[P_{2}\right]$. Therefore, $\operatorname{rem}(H P, N)$ yields a left factor $t-h_{l} \in \mathbb{S}$ of $H P=\left(t-h_{l}\right) P^{\prime}$ according to Lemma 3.6.

Item 1 implies that the Clifford left translations $\eta:[x] \mapsto\left[\left(z-h_{l}\right) x\right]$ and $\bar{\eta}:[x] \mapsto\left[\bar{z}-h_{l}\right]$ are non-singular. By the considerations at the beginning of Sect. 3.3, $\left[P^{\prime}(z)\right]$ and $\left[P^{\prime}(\bar{z})\right]$ are the images of $[H(z) P(z)]$ and $[H(\bar{z}) P(\bar{z})]$ under the inverse Clifford left translations $\eta^{-1}$ and $\bar{\eta}^{-1}$, respectively. 


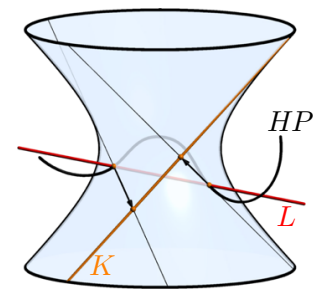

FiguRE 2. Illustration for the choice of $H$ : the non-null line $L:=[H(z) P(z)] \vee[H(\bar{z}) P(\bar{z})]$ is mapped to the right ruling $K$ when dividing off a the left factor $t-h_{l}$. The points $[H(z) P(z)]$ and $[H(\bar{z}) P(\bar{z})]$ are mapped by the respective Clifford left translations

From Item 3 we infer that the left rulings through $[H(z) P(z)]$ and $[H(\bar{z}) P(\bar{z})]$ are different and $\left[P^{\prime}(z)\right] \vee\left[P^{\prime}(\bar{z})\right]$ is either a right ruling of $\mathcal{N}$ or a non-null line. Either way, $\operatorname{rem}\left(P^{\prime}, H H^{*}\right)$ yields a right factor $t-h_{r} \in \mathbb{S}$ of $P^{\prime}=P^{\prime \prime}\left(t-h_{r}\right)$. We define $T_{1}:=H H^{*} \in \mathbb{R}[t]$ and obtain

$$
T_{1} P=\left(t-h^{*}\right)(t-h) P=\left(t-h^{*}\right)\left(t-h_{l}\right) P^{\prime \prime}\left(t-h_{r}\right)
$$

where $\operatorname{deg}\left(P^{\prime \prime}\right)=\operatorname{deg}(P)-1$. We continue by running Algorithm 2 with $P^{\prime \prime}$ and repeat the procedure above whenever the algorithm stops. Finally, we define $T:=T_{1} \ldots T_{m}$ as the product of appearing real polynomials.

It remains to justify the possibility to choose $H$ such that the conditions in Items 1-3 hold.

The a priori degree of freedom for the choice of $H$ is four, namely the four real coefficients of $h$. We will show that each of the conditions in Items $1-3$ accounts for the loss of at most two or three degrees of freedom.

Item 1: $H H^{*} \in \mathbb{R}[t]$ is an irreducible, quadratic polynomial. The number of irreducible, quadratic factors of $P P^{*}$ is finite. Two different irreducible, quadratic real polynomials do not have any common split quaternion zeros (c.f. Lemma 3.4). Hence, our choice of $H$ is restricted by avoiding finitly many zero-sets of quadratic split-quaternions polynomials which are twoparametric, again by Lemma 3.4.

Item 2: Consider the map

$$
\varphi: \mathbb{S} \backslash\left\{g \in \mathbb{S}: g P\left(t_{1}\right)=0\right\} \rightarrow \mathcal{N}: x \mapsto\left[x P\left(t_{1}\right)\right] .
$$

Its image is the left ruling of $\mathcal{N}$ through $\left[P\left(t_{1}\right)\right]$ which also contains $\left[P\left(t_{2}\right)\right]$. By Theorem 2.6, the pre-image of $\left[P\left(t_{2}\right)\right]$ is a one-parametric union of twodimensional affine planes, each of them corresponding to a representative of $\left[P\left(t_{2}\right)\right]$. This is even true for $t_{2} \in \mathbb{C} \backslash \mathbb{R}$ as the subspace of real points in an affine plane of complex dimension two is at most of real dimension two. We choose $H$ such that the split quaternion $H\left(t_{2}\right)^{*} H\left(t_{1}\right) \in \mathbb{S}$ is not contained in the union of these planes. This is possible because $H\left(t_{2}\right)^{*} H\left(t_{1}\right)$ has real split quaternion coefficients

$$
H\left(t_{2}\right)^{*} H\left(t_{1}\right)=\left(t_{2}-h^{*}\right)\left(t_{1}-h\right)=\underbrace{t_{1} t_{2}+h h^{*}}_{\in \mathbb{R}}-\underbrace{\left(t_{1}+t_{2}\right)}_{\in \mathbb{R}} h
$$


and, for varying coefficients of $h$, parametrizes a real four-parametric set in the vector space of real split quaternions. By the choice of $H$, we have $\left[P\left(t_{2}\right)\right] \neq\left[H\left(t_{2}\right)^{*} H\left(t_{1}\right) P\left(t_{1}\right)\right]$ and therefore

$$
\left[H\left(t_{2}\right) P\left(t_{2}\right)\right] \neq\left[H\left(t_{2}\right) H\left(t_{2}\right)^{*} H\left(t_{1}\right) P\left(t_{1}\right)\right]=\left[H\left(t_{1}\right) P\left(t_{1}\right)\right] .
$$

Item 3: By Item 1 , we have $[P(z)],[P(\bar{z})] \notin \mathcal{N}$. The span of $[H(z)]$ and $[H(\bar{z})]$ is a straight line $[H(z)] \vee[H(\bar{z})]$ which is no ruling of $\mathcal{N}$ because $[H(\infty)]=[1] \notin \mathcal{N}$. By Remark 2.5, the point $[H(z) P(z)]$ is contained in the right ruling through $[H(z)]$ and $[H(\bar{z}) P(\bar{z})]$ is contained in the right ruling through $[H(\bar{z})]$. These two rulings are different and hence $[H(z) P(z)] \neq$ $[H(\bar{z}) P(\bar{z})]$. It only remains to ensure that $[H(z) P(z)] \vee[H(\bar{z}) P(\bar{z})]$ is no left ruling of $\mathcal{N}$, i. e.

$$
H(z) P(z)(H(\bar{z}) P(\bar{z}))^{*}=H(z) P(z) P(\bar{z})^{*} H(\bar{z})^{*} \neq 0 .
$$

A direct computation shows that $P(z) P(\bar{z})^{*}$ and $H(z) P(z) P(\bar{z})^{*} H(\bar{z})^{*}$ are of the form

$$
\begin{gathered}
P(z) P(\bar{z})^{*}=P(z) \overline{P(z)^{*}}=p_{0}+\underbrace{\left(p_{1} \mathbf{i}+p_{2} \mathbf{j}+p_{3} \mathbf{k}\right)}_{=: p} \mathbf{i} \neq 0, \\
H(z) P(z) P(\bar{z})^{*} H(\bar{z})^{*}=H(z) P(z) \overline{(H(z) P(z))^{*}}=q_{0}+\left(q_{1} \mathbf{i}+q_{2} \mathbf{j}+q_{3} \mathbf{k}\right) \mathrm{i}
\end{gathered}
$$

where $p_{\ell}, q_{\ell} \in \mathbb{R}$ for $\ell=0, \ldots, 3$ and $\mathrm{i} \in \mathbb{C}$ is the imaginary unit. If $p=0$, then

$$
H(z) P(z) P(\bar{z})^{*} H(\bar{z})^{*}=H(z) p_{0} H(\bar{z})^{*}=p_{0} H(z) H(\bar{z})^{*} \neq 0
$$

since $[H(z)] \vee[H(\bar{z})]$ is no ruling of $\mathcal{N}$. Else, another straightforward computation, which uses the condition $H(z) H(z)^{*}=0$ for simplification, shows that

$$
q_{0}=2 z_{1}\langle h, p\rangle+2 p_{0}+z_{1}^{2},
$$

where $z_{1} \in \mathbb{R}$ is the imaginary part of the complex number $z \in \mathbb{C}$. If $q_{0}=0$, we replace $h$ by another element in the two-parametric set of zeros of $H H^{*}$. This does not change $z, z_{1}$ and $p$. The equation $H H^{*}=0$ defines a quadratic surface in the three-dimensional affine space (c.f. Remark 3.5), given by an equation of the shape $a h_{0}+b=0$ with $a, b \in \mathbb{R}$. Because of $p \neq 0$, it is different from the affine space $\left\{x \in \mathbb{S}: 2 z_{1}\langle x, p\rangle+2 p_{0}+z_{1}^{2}=0\right\}$. Hence, the latter three-space intersects the quadric $H H^{*}=0$ (at most) in a conic section. This shows that there exists a replacement for $h$ such that $q_{0} \neq 0$ and thus $H(z) P(z) P(\bar{z})^{*} H(\bar{z})^{*} \neq 0$. In addition, we can guarantee that the conditions of Items 1 and 2 still hold if we choose our new $h$ near the initial one due to continuity of the polynomial inequalities in Item 1 and 2.

Example 3.18. Consider the polynomial $P_{3}=t^{3}-\mathbf{i} t^{2}+\mathbf{k} t-\mathbf{j}$ from Example 3.15. It does not admit a factorization. Algorithm 2 stops after one iteration for the triple $\left(t^{2}+1, t^{2}-1, t^{2}+1\right)$ and yields only one linear right factor $P_{3}=\left(t^{2}+\mathbf{k}\right)(t-\mathbf{i})$. We define $H=t-2 \mathbf{i} \in \mathbb{S}, T=H H^{*}=t^{2}+4 \in \mathbb{R}[t]$ and obtain the factorization

$$
T P_{3}=(t+2 \mathbf{i})(t-2 \mathbf{i})\left(t^{2}+\mathbf{k}\right)(t-\mathbf{i})
$$




$$
=(t+2 \mathbf{i})\left(t-\frac{3}{4} \mathbf{i}+\frac{5}{4} \mathbf{j}\right)\left(t+\frac{61}{60} \mathbf{i}-\frac{11}{60} \mathbf{j}\right)\left(t-\frac{34}{15} \mathbf{i}-\frac{16}{15} \mathbf{j}\right)(t-\mathbf{i}) .
$$

From a geometric point of view, a split quaternion polynomial $P \in \mathbb{S}[t]$ does neither have a right factor nor a left factor precisely if each quadratic, real factor $N=\left(t-t_{1}\right)\left(t-t_{2}\right)$ of $P P^{*}$ yields a remainder polynomial $R=$ $\operatorname{rem}(P, N)$ that parametrizes only a point of $\mathcal{N}$, i.e. $\left[P\left(t_{1}\right)\right]=\left[P\left(t_{2}\right)\right] \in \mathcal{N}$. Multiplication by the polynomials $H=t-h \in \mathbb{S}$ in the proof of Theorem 3.17 separates these "two" points and in addition guarantees that the line $[H(z) P(z)] \vee[H(\bar{z}) P(\bar{z})]$ does not degenerate to only a point when dividing off the left factor of $H P$ obtained by $N$. Hence, at least one further iteration of Algorithm 2 is successful for $H P$.

Pseudo-code for factorization via multiplying with a real polynomial is provided in Algorithm 3. Lines 1 to 13 are essentially identical to Algorithm 2, the novel part starts with Line 14 .

\subsection{Factorization of Euclidean Motions}

The group of Euclidean motions in three-dimensional Euclidean space can be parametrized by a certain subset of (Hamiltonian) dual quaternions. Hamiltonian quaternions $\mathbb{H}$ are defined in the same manner as split quaternions with only some changes of sign in the generating relations:

$$
\mathbf{i}^{2}=\mathbf{j}^{2}=\mathbf{k}^{2}=\mathbf{i j k}=-1 \text {. }
$$

Unlike the split quaternions, they define a skew field. The non-commutative ring of dual quaternions is defined as $\mathbb{D} \mathbb{H}:=\mathbb{H}[\varepsilon] /\left\langle\varepsilon^{2}\right\rangle$, the quotient of the polynomial ring $\mathbb{H}[\varepsilon]$ and the ideal generated by $\varepsilon^{2}$ where the indeterminate $\varepsilon$ with the property $\varepsilon^{2}=0$ commutes with the complex units $\mathbf{i}, \mathbf{j}$ and $\mathbf{k}$. The norm of a dual quaternion $p+\varepsilon d \in \mathbb{D} \mathbb{H}$ with $p, d \in \mathbb{H}$ equals $(p+\varepsilon d)\left(p^{*}+\right.$ $\left.\varepsilon d^{*}\right)=p p^{*}+\varepsilon\left(p d^{*}+d p^{*}\right)$. It is an element of $\mathbb{R} /\left\langle\varepsilon^{2}\right\rangle-$ the ring of dual numbers. The group of Euclidean motions SE(3) is isomorphic to the quadric defined by the equation $p d^{*}+d p^{*}=0$ minus the subspace $\{p+\varepsilon d \in \mathbb{D H}: p=$ $0\}$ in the seven-dimensional projective space of dual quaternions $\mathbb{P}(\mathbb{D} \mathbb{H})$. A rational motion in the Euclidean three-space can be represented by a dual quaternion polynomial $P+\varepsilon D \in \mathbb{D} \mathbb{H}[t]$ with $P, D \in \mathbb{H}[t]$ that fulfills the Study condition $P D^{*}+D P^{*}=0$ as well as $P \neq 0$. We call such polynomials motion polynomials. Their norm polynomials equal $(P+\varepsilon D)\left(P^{*}+\varepsilon D^{*}\right)=P P^{*}$ and are real. For more details we refer to [9].

Some of our ideas and results can be applied for the factorization of motion polynomials as well and shed new light on the already quite sophisticated Euclidean theory [12]. Consider a reduced motion polynomial $P+\varepsilon D \in \mathbb{D} \mathbb{H}[t]$ and its norm polynomial $(P+\varepsilon D)\left(P^{*}+\varepsilon D^{*}\right)=P P^{*}+\varepsilon\left(P D^{*}+D P^{*}\right)=P P^{*}$. Let $N$ be a quadratic factor of $P P^{*}$. Generically, $R=\operatorname{rem}(P+\varepsilon D, N)$ has a unique left/right zero which yields a linear left/right factor of $P+\varepsilon D$. If $P \in \mathbb{H}[t]$ has a quadratic, real, irreducible factor $M \in \mathbb{R}[t]$ we can choose $N=M$. Then $R$ is of the form $R=\varepsilon R^{\prime}$ for some $R^{\prime} \in \mathbb{H}[t]$ and has no unique zero anymore. This issue has already been addressed in [12] and could be overcome by computing a common quaternion left/right zero of $N$ and $D$ which again yields a linear left/right factor of $P+\varepsilon D$. In the following we will 


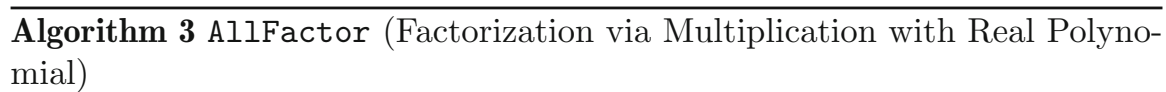

Input: $(P, F, T)$, where $P \in \mathbb{S}[t]$ is a reduced, monic, split quaternion polynomial of degree $n \geq 1, T \in \mathbb{R}[t]$ is a real polynomial (initially $T=1$ ) and $F=\left(F_{1}, \ldots, F_{n}\right)$ is a tuple of quadratic real polynomials such that $P P^{*}=F_{1} \ldots F_{n}$.

Output: A pair $(L, T)$, where $L=\left(L_{1}, \ldots, L_{m}\right)$ is a tuple of linear split quaternion polynomials and $T$ is a real polynomial such that $T P=$ $L_{1} \ldots L_{m}$ with $m \geq n$.

1: If $\operatorname{deg}(P)=0$ Then

2: $\quad$ Return ()

\section{3: End If}

4: $N \leftarrow F_{n}, F \leftarrow\left(F_{1}, \ldots, F_{n-1}\right)$

5: $R=r_{1} t+t_{0} \leftarrow \operatorname{rem}(P, N)$

6: If $R R^{*} \neq 0$ Then

7: $\quad h \leftarrow$ unique right zero of $R$

8: $\quad P \leftarrow \operatorname{lquo}(P, t-h)$

9: $\quad$ Return (ALLFactor $(P, F, T) \oplus(t-h), T)$

10: Else If $r_{1} r_{0}^{*} \neq 0$ Then $\quad \triangleright\left[r_{0}\right] \vee\left[r_{1}\right]$ does not lie on a left ruling

11: $\quad h \leftarrow$ unique common right zero of $N$ and $R \quad \triangleright$ compute via Remark 3.7

12: $\quad P \leftarrow \operatorname{lquo}(P, t-h)$

13: $\quad$ Return $(\operatorname{AllFactor}(P, F, T) \oplus(t-h), T)$

14: Else

15: $\quad$ Choose $H=t-h$ according to Item $1-3$ in the proof of Theorem 3.17.

16: $\quad R_{l} \leftarrow \operatorname{rem}(H P, N)$

17: $\quad h_{l} \leftarrow$ unique common left zero of $N$ and $R_{l} \quad \triangleright$ compute via Remark 3.7

18: $\quad P^{\prime} \leftarrow \operatorname{rquo}\left(H P, t-h_{l}\right)$

19: $\quad R_{r} \leftarrow \operatorname{rem}\left(P^{\prime}, H H^{*}\right)$

20: $\quad h_{r} \leftarrow$ unique common right zero of $H H^{*}$ and $R_{r} \quad \triangleright$ compute via Remark 3.7

21: $\quad P^{\prime \prime} \leftarrow \operatorname{lquo}\left(P^{\prime}, t-h_{r}\right)$

22: $\quad T \leftarrow T H H^{*}$

23: $\quad$ Return $\left(\left(t-h^{*}, t-h_{l}\right) \oplus \operatorname{AllFactor}\left(P^{\prime \prime}, F, T\right) \oplus\left(t-h_{r}\right), T\right)$

24: End If

consider the situation above from a more geometric point of view and compute a common dual quaternion zero of $N$ and $R$. Similar to Lemma 3.4, the set of quaternion zeros of the irreducible, real polynomial $N$ is a Euclidean sphere in some three-dimensional affine space [10].

Let $p \in \mathbb{H}$ be a zero of $N$. A straightforward computation shows that $p+\varepsilon d \in \mathbb{D} \mathbb{H}$ is a zero of $N$ precisely if $d \in \mathbb{H}$ fulfills two linear equations induced by the coefficients of $N$. Hence, the set of dual quaternion zeros of 
$N$ can be viewed as a four-dimensional "cylinder" $C$ in the eight-dimensional vector space $\mathbb{D H}$.

If $\operatorname{deg}(R)=\operatorname{deg}\left(\varepsilon R^{\prime}\right)=1$, then $R^{\prime} \in \mathbb{H}[t]$ has a unique zero in $\mathbb{H}$, let's say $r_{1}$. Moreover, $r_{1}+\varepsilon r_{2} \in \mathbb{D} \mathbb{H}$ is a zero of $R=\varepsilon R^{\prime}$ for any $r_{2} \in \mathbb{H}$. In this case, the set of dual quaternion zeros of $R$ is a four-dimensional affine subspace $A$ of $\mathbb{D H}$.

We are interested in motion polynomial factorizations. Hence, the Study condition $\left(t-r_{1}-\varepsilon r_{2}\right)\left(t-r_{1}{ }^{*}-\varepsilon r_{2}{ }^{*}\right) \in \mathbb{R}[t]$ should hold. It induces two linear constraints on $r_{2}$, namely $r_{1} r_{2}{ }^{*}+r_{2} r_{1}{ }^{*}=0$ and $r_{2}+r_{2}{ }^{*}=0$. Hence, $\varepsilon r_{2}$ lies in a two-dimensional affine subspace $B$ of $\varepsilon \mathbb{H} \subset \mathbb{D} \mathbb{H}$.

The intersection of $A$ and $C$ yields all common dual quaternion left/right zeros of $N$ and $R$ and thus linear left/right factors of $P+\varepsilon D$. Intersecting with $B$ imposes the motion polynomial condition. If this intersection is not empty (which is not guaranteed) we might even find new factorizations for certain dual quaternion polynomials.

Example 3.19. Consider the polynomial $P_{4}=\left(t^{2}+1\right)(t-\mathbf{k})-\varepsilon\left(\mathbf{i} t^{2}+(\mathbf{i}+\right.$ $\mathbf{j}) t+\mathbf{j})$, a special case of $[12$, Example 5]. The norm polynomial reads as $P_{4} P_{4}{ }^{*}=\left(t^{2}+1\right)^{3}$. We can only choose the factor $N=t^{2}+1$ and compute $R=\operatorname{rem}\left(P_{4}, N\right)=-\varepsilon((\mathbf{i}+\mathbf{j}) t-\mathbf{i}+\mathbf{j})$. The set $A \cap B$ of its left zeros fulfilling the Study condition is $\{-\mathbf{k}+\varepsilon(\lambda \mathbf{i}+\mu \mathbf{j}): \lambda, \mu \in \mathbb{R}\}$. The set $C$ of left zeros of $N$ equals

$$
C=\left\{\alpha \mathbf{i}+\beta \mathbf{j}+\gamma \mathbf{k}+\varepsilon(\lambda \mathbf{i}+\mu \mathbf{j}): \alpha, \beta, \gamma, \lambda, \mu \in \mathbb{R} \text { and } \alpha^{2}+\beta^{2}+\gamma^{2}=1\right\} .
$$

Hence, $H=t+\mathbf{k}+\varepsilon(\lambda \mathbf{i}+\mu \mathbf{j}) \in \mathbb{D} \mathbb{H}[t]$ is a left motion polynomial factor of $P_{4}$ for all $\lambda, \mu \in \mathbb{R}$. The right quotient $\operatorname{rquo}\left(P_{4}, H\right)=t^{2}-2 \mathbf{k} t-1+\varepsilon((\lambda \mathbf{i}+$ $\mu \mathbf{j}-\mathbf{i}) t-\mu \mathbf{i}+\lambda \mathbf{j}-\mathbf{i})$ can be factored by the generic factorization algorithm and we obtain $P_{4}=H_{1} H_{2} H_{3}$ with

$$
\begin{aligned}
& H_{1}=t+\mathbf{k}+\varepsilon(\lambda \mathbf{i}+\mu \mathbf{j}), \\
& H_{2}=t-\mathbf{k}+\varepsilon\left(\left(\lambda-\frac{1}{2}\right) \mathbf{i}+\left(\mu+\frac{1}{2}\right) \mathbf{j}\right), \\
& H_{3}=t-\mathbf{k}-\varepsilon\left(\frac{1}{2} \mathbf{i}+\frac{1}{2} \mathbf{j}\right),
\end{aligned}
$$

for all $\lambda, \mu \in \mathbb{R}$.

Remark 3.20. Our approach yields a systematic way to compute new factorizations for certain motion polynomials. Indeed, the leftmost linear factor in all factorizations of $P_{4}$ from Example 3.19 in [12] are quaternion factors while ours are proper dual quaternion factors.

\section{Future Research}

We have presented a new algorithm for factorization of split quaternion polynomials. Its failure is equivalent to the non-factorizability of the polynomial into linear factors. By investigation on the algorithm's geometric behavior, we presented a procedure in order to factor all motions in the hyperbolic plane represented by split quaternion polynomials. 
Unfortunately, a test for factorizability of split quaternion polynomials using our characterization is of factorial complexity in the polynomial degree as we might need to run Algorithm 2 for all possible tuples of quadratic, real factors of the norm polynomial. An a priori characterization as well as a numerically stable and robust version of Algorithm 2 is on our research agenda.

Our approach seems promising to find all possible factorizations into linear factors of dual quaternion polynomials as well, a question which is also still open yet. Further plans for future research include extensions of polynomial factorization in the more general setup of Geometric Algebra. It admits a conjugation, an important ingredient for the factorization algorithm but the corresponding norm is not multiplicative which might cause problems. One particularly interesting example is conformal geometric algebra for which promising preliminary results do exist. The description in $[5,6]$ of the exponential in conformal geometric algebra allows for a kinematic interpretation of factorization, similar to hyperbolic kinematics (Sect. 2.2).

\section{Acknowledgements}

Daniel F. Scharler was supported by the Austrian Science Fund (FWF): P 31061 (The Algebra of Motions in 3-Space).

Funding Open access funding provided by University of Innsbruck and Medical University of Innsbruck.

Open Access. This article is licensed under a Creative Commons Attribution 4.0 International License, which permits use, sharing, adaptation, distribution and reproduction in any medium or format, as long as you give appropriate credit to the original author(s) and the source, provide a link to the Creative Commons licence, and indicate if changes were made. The images or other third party material in this article are included in the article's Creative Commons licence, unless indicated otherwise in a credit line to the material. If material is not included in the article's Creative Commons licence and your intended use is not permitted by statutory regulation or exceeds the permitted use, you will need to obtain permission directly from the copyright holder. To view a copy of this licence, visit http:// creativecommons.org/licenses/by/4.0/.

Publisher's Note Springer Nature remains neutral with regard to jurisdictional claims in published maps and institutional affiliations.

\section{References}

[1] Abrate, M.: Quadratic formulas for generalized quaternions. J. Algebra Appl. 8(3), 289-306 (2009)

[2] Alkhaldi, A.H., Alaoui, M.K., Khamsi, M.A.: New Trends in Analysis and Geometry. Cambridge Scholars Publishing (2020). http://www. cambridgescholars.com/new-trends-in-analysis-and-geometry

[3] Cao, W.: Quadratic formulas for split quaternions (2019). arXiv: 1905.08153 
[4] Casas-Alvero, E.: Analytic Projective Geometry. European Mathematical Society, Zurich (2014)

[5] Dorst, L.: The construction of 3D conformal motions. Math. Comput. Sci. 10, 97-113 (2016). https://doi.org/10.1007/s11786-016-0250-8

[6] Dorst, L., Valkenburg, R.: Square root and logarithm of rotors in 3D conformal geometric algebra using polar decomposition. In: Dorst, L., Lasenby, J. (eds.) Guide to Geometric Algebra in Practice, pp. 81-104. Springer, Berlin (2011). https://doi.org/10.1007/978-0-85729-811-9_5

[7] Falcão, M.I., Miranda, F., Severino, R., Soares, M.J.: The number of zeros of unilateral polynomials over coquaternions revisited. Linear Multilinear Algebra 67(6), 1231-1249 (2019). https://doi.org/10.1080/03081087.2018.1450828

[8] Gordon, B., Motzkin, T.S.: On the zeros of polynomials over division rings. Trans. Am. Math. Soc. 116, 218-226 (1965)

[9] Hegedüs, G., Schicho, J., Schröcker, H.P.: Factorization of rational curves in the study quadric and revolute linkages. Mech. Mach. Theory 69(1), 142-152 (2013). https://doi.org/10.1016/j.mechmachtheory.2013.05.010

[10] Huang, L., So, W.: Quadratic formulas for quaternions. Appl. Math. Lett. 15(15), 533-540 (2002)

[11] Li, Z., Scharler, D.F., Schröcker, H.P.: Factorization results for left polynomials in some associative real algebras: state of the art, applications, and open questions. J. Comput. Appl. Math. 349, 508-522 (2019). https://doi.org/10. 1016/j.cam.2018.09.045

[12] Li, Z., Schicho, J., Schröcker, H.P.: Factorization of motion polynomials. J. Symb. Comput. 92, 190-202 (2019). https://doi.org/10.1016/j.jsc.2018.02.005

[13] Li, Z., Schicho, J., Schröcker, H.P.: The geometry of quadratic quaternion polynomials in Euclidean and non-Euclidean planes. In: L. Cocchiarella (ed.) ICGG 2018-Proceedings of the 18th International Conference on Geometry and Graphics, pp. 298-309. Springer International Publishing, Cham (2019)

[14] Niven, I.: Equations in quaternions. Am. Math. Mon. 48(10), 654-661 (1941)

[15] Scharler, D.F., Siegele, J., Schröcker, H.P.: Quadratic split quaternion polynomials: factorization and geometry. Adv. Appl. Clifford Algebras 30, 23 (2019). https://doi.org/10.1007/s00006-019-1037-1

[16] Siegele, J., Scharler, D.F., Schröcker, H.P.: Rational motions with generic trajectories of low degree. Comput. Aided Geom. Design 76, 101793 (2020). https://doi.org/10.1016/j.cagd.2019.101793

[17] Wildberger, N.: Universal hyperbolic geometry II: a pictorial overview. KoG 14, 3-24 (2010)

[18] Wildberger, N.: Universal hyperbolic geometry III: first steps in projective triangle geometry. KoG 15, 25-49 (2011)

[19] Wildberger, N.: Universal hyperbolic geometry I: trigonometry. Geom. Dedicata 163, 215-274 (2013). https://doi.org/10.1007/s10711-012-9746-9 
Daniel F. Scharler and Hans-Peter Schröcker

Department of Basic Sciences in Engineering Sciences

University of Innsbruck

Technikerstr. 13

6020 Innsbruck

Austria

e-mail: daniel.scharler@uibk.ac.at

Hans-Peter Schröcker

e-mail: hans-peter.schroecker@uibk.ac.at

Received: October 12, 2020.

Accepted: March 12, 2021. 